\title{
OPEN Prevalence and risk factors for faecal carriage of multidrug resistant Escherichia coli among slaughterhouse workers
}

\author{
Mabel Kamweli Aworh ${ }^{1,2}$, Oluwadamilola Abiodun-Adewusi ${ }^{2,3}$, Nwando Mba ${ }^{4}$, \\ Birgitte Helwigh ${ }^{5}$ \& Rene S. Hendriksen ${ }^{6}$
}

The increasing occurrence of antimicrobial-resistant Escherichia coli in human and animal population has become a global public health problem that requires immediate intervention. We aimed to investigate prevalence and risk factors for faecal carriage of drug-resistant $E$. coli among slaughterhouse workers. We conducted this cross-sectional study among 118 apparently healthy workers in the largest slaughterhouses in Abuja and Lagos from July to December 2020. $E$. coli was isolated from stool samples of slaughterhouse workers and antimicrobial susceptibility testing performed using the Kirby-Bauer disk diffusion method. Multi-drug resistance (MDR) was defined as resistance to three or more classes of antibiotics. Majority were males: $88.1 \%(n=104)$, aged $>41$ years: $28.8 \%(n=34)$, married: $70.3 \%(n=83)$, and were butchers: $53.4 \%(n=63)$. Prevalence of MDR E. coli was $50 \%(n=59)$, highest among butchers compared to slaughterhouse cleaners. Of $75 \mathrm{E}$. coli isolates identified, $25.3 \%(n=19)$ were ESBL producers; $78.7 \%(n=59)$ were MDR. Keeping animals $(p=0.01)$; eating at the slaughterhouse $(p=0.03)$ and collecting waste $(p=0.02)$ remained independent risk factors for acquiring MDR $E$. coli. Prevalence of resistant $E$. coli was highest among butchers and associated with keeping animals at home, eating at work, and waste-collection. Handhygiene and responsible use of antibiotics among slaughterhouse workers should be encouraged.

Globally, Escherichia coli, which is known as an indicator bacteria, is a common source of foodborne illnesses especially in sub-Saharan Africa including Nigeria ${ }^{1,2}$. The emergence of multi-drug resistant (MDR) foodborne pathogens has further worsened the public health implications of antimicrobial resistance (AMR) resulting in increased length of hospital stay, mortality, and economic burden ${ }^{3}$. Sub-Saharan Africa, particularly Nigeria is not exempted from the challenges posed by $\mathrm{AMR}^{1,2}$ as a review conducted in West Africa reported a majority of the studies were from Nigeria ${ }^{4}$. The World Health Organization (WHO) has reported that a high level of AMR observed in the human population is due to misuse or abuse of antimicrobials in the food and agriculture sectors ${ }^{5}$.

Food, particularly meat is a major source of MDR-E. coli among humans ${ }^{6,7}$. In many developing economies, food-producing animals are slaughtered and processed under very poor hygienic conditions ${ }^{8}$, hence, constituting an occupational risk to slaughterhouse workers (SHWs). MDR-E. coli, often detected in the guts of apparently healthy individuals, may not directly cause disease, but serve as a reservoir of AMR genes ${ }^{9,10}$. These resistant genes can further spread to other Gram-negative pathogenic bacteria in the gut and potentially become harmful hence causing disease but can also spread due to open defecation and poor hygiene ${ }^{11}$.

Slaughterhouses in some settings are hotspots for MDR pathogens, facilitating their spread to the environment ${ }^{12}$. Evidence shows that SHWs are at risk of being exposed to certain bacterial infections because of their occupation ${ }^{13,14}$. Antimicrobial drugs (AMDs) are also easily accessible to livestock farmers without any prescription from the veterinarian, thus, encouraging antimicrobial use (AMU) indiscriminately in foodproducing animals in Nigeria due to easy access ${ }^{15}$.

\footnotetext{
${ }^{1}$ Department of Veterinary and Pest Control Services, Federal Ministry of Agriculture and Rural Development, Abuja, Nigeria. ${ }^{2}$ Nigeria Field Epidemiology and Laboratory Training Programme, Abuja, Nigeria. ${ }^{3}$ National Agency for Food and Drug Administration and Control (NAFDAC), Lagos, Nigeria. ${ }^{4}$ National Reference Laboratory, Nigeria Center for Disease Control, Abuja, Nigeria. ${ }^{5}$ Department for Global Surveillance, National Food Institute, Technical University of Denmark, Kgs. Lyngby, Denmark. ${ }^{6} \mathrm{WHO}, \mathrm{FAO}, \mathrm{EU}$, Reference Laboratory for Antimicrobial Resistance, National Food Institute, Technical University of Denmark, Kgs. Lyngby, Denmark. ${ }^{\square}$ email: mabelaworh@yahoo.com
} 
Few studies conducted in Nigeria have examined the presence of MDR-E.coli in human patients and foodproducing animals, however, the risk factors for faecal carriage of MDR-E. coli among SHWs is not yet known ${ }^{16,17}$. A recent review has reported that human exposure to environmental sources was an important risk factor for the acquisition of MDR-E. coli ${ }^{10}$. Most slaughterhouse environments in Nigeria are poorly managed with very poor sanitary conditions, hence promoting the spread of resistant pathogens ${ }^{18}$. Nigeria has a growing population of livestock characterized by an extensive system of management making it difficult to track AMU in this food-animal population because of their constant movement in search of food. Besides, there are problems of illegal importation and sale of antimicrobials for use in livestock ${ }^{19}$. It is also practically impossible to estimate the public health risks encountered by SHWs when they share the same environment with livestock and their waste products ${ }^{2}$.

There is increased AMU among livestock in Nigeria with a resultant increase in the development of AMR among enteric pathogens and indicator bacteria such as E. coli. It is therefore imperative to generate data on the resistance profile of indicator bacteria among apparently healthy individuals exposed to livestock by their occupation for proper diagnosis and treatment of bacterial infections.

We hypothesized that livestock harboring MDR-E. coli facilitates the transmission of the pathogen to workers exposed to livestock and the slaughterhouse environment. This study aimed to investigate the prevalence and risk factors for faecal carriage of MDR-E. coli among SHWs to generate baseline data for the establishment of the AMR Animal-Health Surveillance system in Nigeria. Prevalence was determined by antimicrobial susceptibility testing while the risk was accessed based on interviews and multivariate analysis.

\section{Results}

Of the 118 SHWs interviewed, majority were males: $88.1 \%(\mathrm{n}=104)$, aged $>41$ years: $28.8 \%(\mathrm{n}=34)$, married: $70.3 \%(n=83)$, Muslims: $70.3 \%(n=83)$, had Islamic education: $31.4 \%(n=37)$, lived in urban areas: $96.6 \%$ $(n=114)$, of Hausa ethnicity: $73.7 \%(n=87)$ and were butchers: $53.4 \%(n=63)$ (Table 1$)$.

Prevalence of MDR E. coli among slaughterhouse workers. Fifty-nine SHWs of the 118 included in the study tested positive for MDR-E. coli giving a prevalence of $50 \%$. A majority of these, $69.5 \%(\mathrm{n}=41)$ resided in Abuja and the highest prevalence of $50.9 \%$ was among butchers (prevalence $O R=1.00,95 \%$ confidence interval $[\mathrm{CI}]=0.37-2.69 ; \mathrm{p}=0.00)$ when compared to slaughterhouse cleaners.

Out of 75 E. coli isolates identified, $25.3 \%(n=19)$ were ESBL-E. coli while $78.7 \%(\mathrm{n}=59)$ were MDR. The isolates were resistant to ciprofloxacin: $(81.3 \%, n=61)$, tetracycline: $(77.3 \%, n=58)$, sulfonamides: $(72 \%, n=54)$, trimethoprim: $(72 \%, \mathrm{n}=54)$, ampicillin: $(72 \%, \mathrm{n}=54)$, nalidixic acid: $(57.3 \%, \mathrm{n}=43)$, cefotaxime: $(25.3 \%, \mathrm{n}=19)$, gentamicin: $(20 \%, \mathrm{n}=15)$, chloramphenicol: $(13.3 \%, \mathrm{n}=10)$, ceftazidime: $(13.3 \%, \mathrm{n}=10)$, and azithromycin: $(10.7 \%, \mathrm{n}=8)$ (Fig. 1).

All the E. coli isolates were $100 \%$ susceptible to cefoxitin, colistin, imipenem, meropenem, and nitrofurantoin. The maximum number of ESBL-E. coli was isolated from butchers: $57.9 \%(\mathrm{n}=11)$ when compared to other SHWs. The resistance rates of the E. coli isolates to ciprofloxacin, ampicillin, tetracycline, sulfonamides, trimethoprim, nalidixic acid, cefotaxime, and chloramphenicol were higher among butchers when compared to the rest of the SHWs. All the isolates were, however, sensitive to cefoxitin, colistin, imipenem, meropenem, and nitrofurantoin (Table 2).

In this study, $6.6 \%(n=5)$ of the isolates were susceptible to all the AMDs tested, $4 \%(n=3)$ were resistant to only one AMD while $9.3 \%(n=7)$ were resistant to only two AMDs. Surprisingly one isolate was resistant to 11 different AMDs including those of critical importance for human health. In $72 \%(n=54)$ of the isolates, a MAR index greater than 0.2 was observed (Fig. 2).

Among the 19 ESBL-E. coli isolates, high resistance was observed for cefotaxime: $100 \%(\mathrm{n}=19)$, ciprofloxacin: 94.7\% ( $=18)$, tetracycline: $94.7 \%(n=18)$, sulfonamides: $94.7 \%(n=18)$, trimethoprim: $94.7 \%(n=18)$, ampicillin: $94.7 \%(n=18)$ and nalidixic acid: $78.9 \%(n=15)$.

Antimicrobial use patterns of slaughterhouse workers. Among 59 SHWs colonized with MDR-E. coli, $61 \%(\mathrm{n}=36)$ reported that they had taken antibiotics in the last month before stool collection. Less than half $39 \%(n=23)$ reported that the last antibiotics they had taken were Ampiclox (a combination of ampicillin and cloxacillin) especially when they had a knife cut. About a third $35.6 \%(n=21)$ of the workers reported that they self-prescribed antimicrobials more than three times in the last three months before sampling. A majority $93.2 \%$ $(n=55)$ reported that they usually purchased antibiotics from a chemist although $89.8 \%(n=53)$ reported that they usually received direction for use from the pharmacist. More than two-thirds $71.2 \%(n=42)$ reported that they usually completed their dosage while $30.5 \%(n=18)$ reported that they stopped their medication whenever they felt better.

Risk factors for MDR-E. coli among SHWs. Occupational hazards reported among the SHWs were quite a few. Nearly half $(49.2 \% ; n=58)$ of the workers reported that a major hazard they faced was knife cuts while slaughtering the animals. Over half $(56.9 \% ; n=33)$ reported that they however had to continue processing slaughtered animals even after sustaining a knife cut, hence their wounds get exposed to infection. Nearly half $(48.3 \% ; \mathrm{n}=57)$ reported that they often handle fetuses recovered from slaughtered pregnant animals.

The factors associated with MDR-E. coli among SHWs were: aged over 41 years old (OR 2.79, 95\% CI 1.21-6.45); keeping animals at home (OR 2.13, CI 1.02-4.44); eating at the slaughterhouse while working (OR 2.13, CI 1.02-4.44); collecting slaughterhouse waste (OR 6.53, 95\% CI 1.38-30.92); and washing hands with soap (OR 0.42, 95\% CI 0.19-0.93) (Table 3). 


\begin{tabular}{|c|c|c|}
\hline Variables & $\mathbf{n}$ & $\%$ \\
\hline \multicolumn{3}{|l|}{ Age group (years) } \\
\hline $18-25$ & 23 & 19.5 \\
\hline $26-33$ & 29 & 24.6 \\
\hline $34-41$ & 32 & 27.1 \\
\hline$>41$ & 34 & 28.8 \\
\hline \multicolumn{3}{|l|}{ Marital status } \\
\hline Divorced & 1 & 0.9 \\
\hline Married & 66 & 54.6 \\
\hline Single & 55 & 45.5 \\
\hline Widowed & 2 & 1.7 \\
\hline \multicolumn{3}{|l|}{ Educational level } \\
\hline None & 6 & 5.1 \\
\hline Islamic education & 37 & 31.4 \\
\hline Primary & 17 & 14.4 \\
\hline Secondary & 34 & 28.8 \\
\hline Tertiary & 24 & 20.3 \\
\hline \multicolumn{3}{|l|}{ Occupation } \\
\hline Butchers & 63 & 53.4 \\
\hline Slaughterhouse cleaners & 21 & 17.8 \\
\hline Vets/para-vets & 11 & 9.3 \\
\hline Meat carriers & 10 & 8.5 \\
\hline Meat sellers & 6 & 5.1 \\
\hline Livestock traders & 5 & 4.2 \\
\hline Security & 2 & 1.7 \\
\hline \multicolumn{3}{|l|}{ Duration of work } \\
\hline $0-5$ years & 35 & 29.7 \\
\hline$\geq 6$ years & 83 & 70.3 \\
\hline \multicolumn{3}{|l|}{ Location } \\
\hline Abuja & 84 & 71.2 \\
\hline Lagos & 34 & 28.8 \\
\hline \multicolumn{3}{|l|}{ Residence } \\
\hline Rural & 4 & 3.4 \\
\hline Urban & 114 & 96.6 \\
\hline \multicolumn{3}{|l|}{ Religion } \\
\hline Christianity & 35 & 29.7 \\
\hline Islam & 83 & 70.3 \\
\hline \multicolumn{3}{|l|}{ Ethnicity } \\
\hline Ibo & 4 & 3.4 \\
\hline Hausa & 87 & 73.7 \\
\hline Yoruba & 18 & 15.3 \\
\hline Others & 9 & 7.6 \\
\hline
\end{tabular}

Table 1. Demographic characteristics of slaughterhouse workers in Abuja and Lagos-Nigeria, 2020.

Five factors that were statistically significant at bivariate analysis $(\mathrm{p} \leq 0.05)$ were included in the unconditional logistic regression model used in this study. These factors include age over 41 years, keeping animals at home, eating at the slaughterhouse while working, collecting slaughterhouse waste, and washing hands with soap. There were fewer female workers hence gender was considered as a potential confounder and included in the final model used for analysis. Using a stepwise elimination approach and controlling for gender, all factors remained statistically significant in the final logistic regression model. We identified four independent risk factors and one protective factor. Being $\geq 41$ years (adjusted OR $[A O R]=4.47,95 \%$ CI 1.59-12.60); keeping animals at home; $(\mathrm{AOR}=4.04,95 \% \mathrm{CI} 1.48-11.05)$; eating at the slaughterhouse $(\mathrm{AOR}=3.01,95 \% \mathrm{CI} 1.14-7.91)$ and collecting slaughterhouse waste $(\mathrm{AOR}=10.31,95 \% \mathrm{CI} 1.54-69.10)$ remained independent risk factors for faecal carriage of MDR-E. coli among SHWs. Washing hands with soap (AOR $=2.72,95 \%$ CI 1.06-6.96), however, remained a protective factor against MDR-E. coli among SHWs (Table 4).

Unconditional logistic regression analysis showed that SHWs had 4 times the odds of shedding MDR-E. coli in their stool if they were $\geq 41$ years of age $(p=0.01)$, kept animals at home $(p=0.01)$, and 3 times more likely if they ate at the slaughterhouse while processing slaughtered cattle $(\mathrm{p}=0.03)$. Workers were 10 times more likely to 


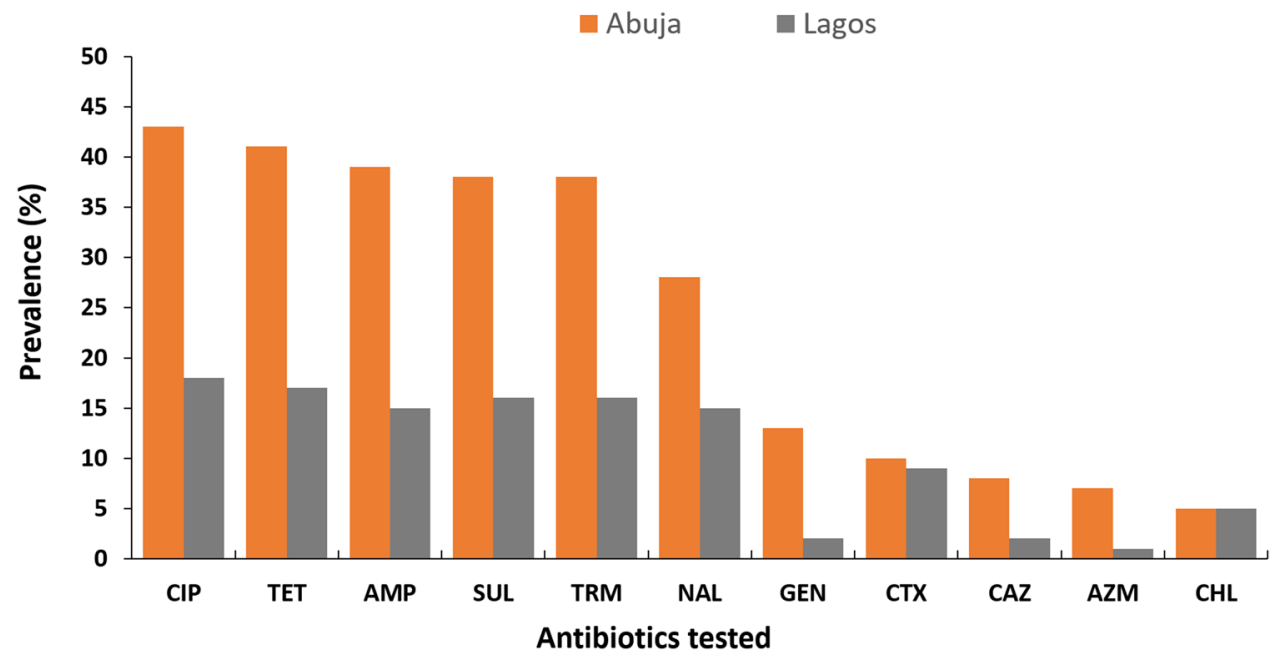

Figure 1. Prevalence of MDR E. coli isolated from stool samples of slaughterhouse workers in Abuja and Lagos slaughterhouses, 2020. Bars represent the proportion of samples containing at least one MDR E. coli isolate with 95\% confidence intervals. Data were obtained from two sources: Abuja and Lagos slaughterhouses. AMP ampicillin, $A Z M$ azithromycin, $C A Z$ ceftazidime, $C T X$ cefotaxime, $C H L$ chloramphenicol, $C I P$ ciprofloxacin, GEN gentamicin, NAL nalidixic acid, SUL sulfonamides, TET tetracycline, and TRM trimethoprim.

\begin{tabular}{|c|c|c|c|c|c|}
\hline Drug class & Drug & $\begin{array}{l}\text { Resistance } \\
\text { breakpoints, } \mathrm{mm}\end{array}$ & Overall, $n=75(\%)$ & Butchers, $n=42(\%)$ & Others, $n=33(\%)$ \\
\hline \multirow{2}{*}{ Quinolones } & Ciprofloxacin $(5 \mu \mathrm{g})$ & $\leq 20$ & $61(81.3)$ & $32(76.2)$ & $29(87.9)$ \\
\hline & Nalidixic acid $(30 \mu \mathrm{g})$ & $\leq 13$ & $43(57.3)$ & $22(52.4)$ & $21(63.6)$ \\
\hline Tetracyclines & Tetracycline $(30 \mu \mathrm{g})$ & $\leq 11$ & $58(77.3)$ & $30(71.4)$ & $28(84.9)$ \\
\hline \multirow{2}{*}{$\begin{array}{l}\text { Folate pathway } \\
\text { antagonists }\end{array}$} & Sulfonamides $(300 \mu \mathrm{g})$ & $\leq 12$ & $54(72.0)$ & $28(66.7)$ & $26(78.8)$ \\
\hline & Trimethoprim $(5 \mu \mathrm{g})$ & $\leq 10$ & $54(72.0)$ & $28(66.7)$ & $26(78.8)$ \\
\hline Penicillins & Ampicillin $(10 \mu \mathrm{g})$ & $\leq 13$ & $54(72.0)$ & $32(76.2)$ & $22(66.7)$ \\
\hline \multirow{2}{*}{ Cephalosporins } & Cefotaxime $(30 \mu \mathrm{g})$ & $\leq 22$ & $19(25.3)$ & $11(26.2)$ & $8(24.2)$ \\
\hline & Ceftazidime $(30 \mu \mathrm{g})$ & $\leq 17$ & $10(13.3)$ & $5(11.9)$ & $5(15.2)$ \\
\hline Aminoglycosides & Gentamicin $(10 \mu \mathrm{g})$ & $\leq 12$ & $15(20.0)$ & $5(11.9)$ & $10(30.3)$ \\
\hline Phenicols & $\begin{array}{l}\text { Chloramphenicol } \\
(30 \mu \mathrm{g})\end{array}$ & $\leq 12$ & $10(13.3)$ & $6(14.3)$ & $4(12.1)$ \\
\hline Macrolides & Azithromycin $(15 \mu \mathrm{g})$ & $\leq 12$ & $8(10.7)$ & $3(7.2)$ & $5(15.2)$ \\
\hline $\begin{array}{l}\text { Resistance to } 3 \text { or } \\
\text { more classes of anti- } \\
\text { biotics }\end{array}$ & MDR & $\mathrm{n} / \mathrm{a}$ & $59(78.7)$ & $30(71.4)$ & $29(87.9)$ \\
\hline
\end{tabular}

Table 2. Resistance profile of E. coli isolates recovered from slaughterhouse workers at Abuja and Lagos slaughterhouses to the antibiotics tested.

shed MDR-E. coli in their stool if they collected slaughterhouse waste $(\mathrm{p}=0.02)$. They were 3 times more likely to be protected from being colonized by MDR-E. coli if they washed their hands with soap and clean water $(p=0.04)$.

\section{Discussion}

To the best of our knowledge, this is the first study to document the prevalence and risk factors for faecal carriage of MDR-E. coli among SHWs in Abuja and Lagos, Nigeria. The present study recorded a high prevalence of $50 \%$ for MDR-E. coli among SHWs in both locations when compared with $36.6 \%$ and $36.8 \%$ observed in the general population respectively ${ }^{23,24}$. A similar study conducted among poultry workers in Abuja reported a lower prevalence of $39.7 \%$ for MDR-E. coli ${ }^{9}$. The high prevalence observed in the present study might be due to AMU in animals which have been associated with the development of bacterial resistance in humans exposed to these food-producing animals ${ }^{25-27}$. The present study demonstrates that $72 \%$ of MDR-E. coli recovered from the SHWs had a MAR index above 0.2. This implies that the isolates may have been recovered from sources with a high frequency of $\mathrm{AMU}^{28}$ although multiple $\mathrm{AMR}$ is usually detected when plasmids encode AMR phenotype ${ }^{22}$.

Our findings show a high prevalence of MDR-E. coli but a low prevalence of ESBL-E. coli colonization in the study population possibly because ceftazidime and cefotaxime are not some of the commonly self-prescribed antimicrobials in the present study. This supports the findings from a survey in the Mekong Delta in Vietnam 


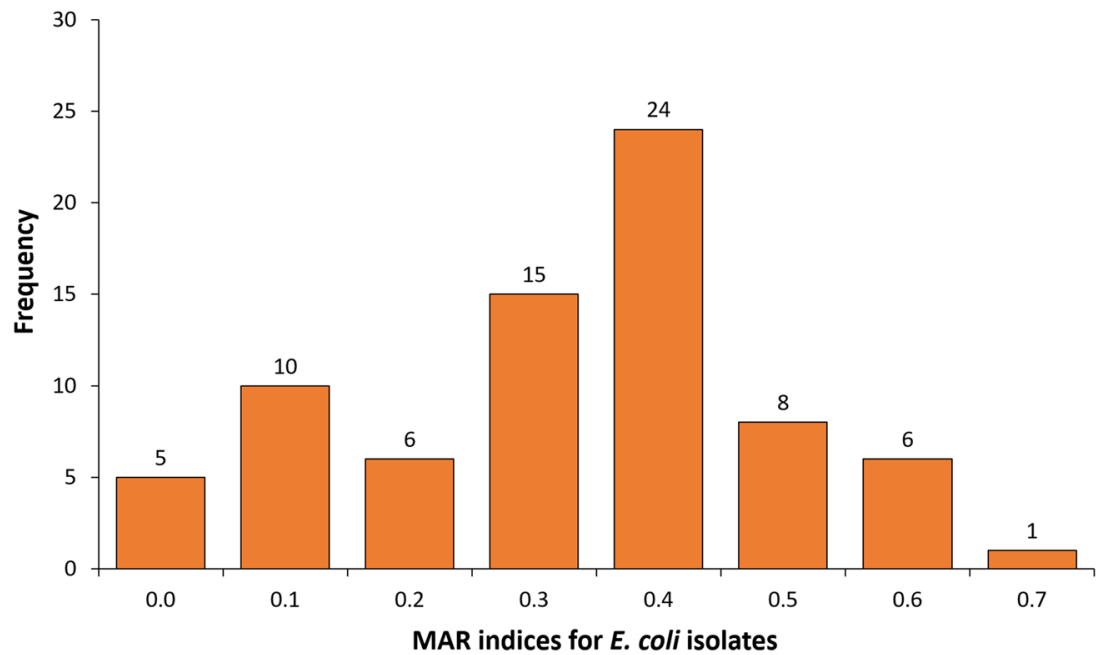

Figure 2. Multiple antibiotics resistance (MAR) indices for E. coli recovered from Slaughterhouse workers in Abuja and Lagos-Nigeria, 2020. This bar chart demonstrates the increase in MAR indices observed for the $E$. coli isolates. Each bar represents the frequency recorded for the different MAR indices. The highest frequency $(\mathrm{n}=24)$ was recorded against a MAR index of 0.4.

\begin{tabular}{|c|c|c|c|c|}
\hline Work exposure & MDR (Yes) & MDR (No) & Odds ratio ( $95 \%$ confidence level) & p value \\
\hline \multicolumn{5}{|l|}{ Age } \\
\hline$\geq 41$ years & 23 & 11 & $2.79(1.21-6.45)$ & $0.02^{*}$ \\
\hline $18-41$ years & 36 & 48 & & \\
\hline \multicolumn{5}{|l|}{ Level of education } \\
\hline Less than secondary school & 29 & 31 & $0.87(0.42-1.80)$ & 0.71 \\
\hline Secondary and above & 30 & 28 & & \\
\hline \multicolumn{5}{|l|}{ Occupation } \\
\hline Butcher & 30 & 33 & $0.82(0.40-1.68)$ & 0.58 \\
\hline Others & 29 & 26 & & \\
\hline \multicolumn{5}{|l|}{ Work exposure (years) } \\
\hline$\geq 6$ & 41 & 42 & $0.92(0.42-2.03)$ & 0.84 \\
\hline $1-5$ & 18 & 17 & & \\
\hline \multicolumn{5}{|c|}{ Slaughtering animal after sustaining an injury } \\
\hline Yes & 18 & 15 & $1.80(0.63-5.16)$ & 0.27 \\
\hline No & 10 & 15 & & \\
\hline \multicolumn{5}{|l|}{ Keeping animals at home } \\
\hline Yes & 34 & 23 & $2.13(1.02-4.44)$ & $0.04^{*}$ \\
\hline No & 25 & 36 & & \\
\hline \multicolumn{5}{|c|}{ Eating while working at slaughterhouse } \\
\hline Yes & 36 & 25 & $2.13(1.02-4.44)$ & $0.04^{\star}$ \\
\hline No & 23 & 34 & & \\
\hline \multicolumn{5}{|c|}{ Collecting slaughterhouse waste } \\
\hline Yes & 11 & 2 & $6.53(1.38-30.92)$ & $0.01^{\star}$ \\
\hline No & 48 & 57 & & \\
\hline \multicolumn{5}{|l|}{ Washing hands with soap } \\
\hline Yes & 19 & 34 & $0.42(0.19-0.93)$ & $0.03^{\star}$ \\
\hline No & 28 & 21 & & \\
\hline \multicolumn{5}{|l|}{ Diarrhoea in last 3 months } \\
\hline Yes & 23 & 27 & $0.76(0.36-1.57)$ & 0.46 \\
\hline No & 36 & 32 & & \\
\hline
\end{tabular}

Table 3. Bivariate analysis of factors associated with multidrug-resistant E. coli recovered from slaughterhouse workers in Abuja and Lagos-Nigeria, 2020. ${ }^{*}$ Values that were significant at bivariate analysis. 


\begin{tabular}{|l|l|l|l|}
\hline Work exposure factors & Adjusted odds ratio & 95\% confidence interval & p value \\
\hline Age $>41$ years & 4.47 & $1.59-12.60$ & $0.01^{*}$ \\
\hline Keeping animals at home & 4.04 & $1.48-11.05$ & $0.01^{\star}$ \\
\hline Eating at the slaughterhouse while working & 3.01 & $1.14-7.91$ & $0.03^{*}$ \\
\hline Collecting slaughterhouse waste & 10.31 & $1.54-69.10$ & $0.02^{\star}$ \\
\hline Washing hands with soap & 2.72 & $1.06-6.96$ & $0.04^{*}$ \\
\hline
\end{tabular}

Table 4. Factors found to be significantly associated with MDR E. coli among slaughterhouse workers in Abuja and Lagos, Nigeria 2020 in a logistic regression model. ${ }^{\star}$ Independent risk factors for MDR E. coli that remained significant in the logistic regression model. ${ }^{\#}$ Only protective factor against MDR E. coli that was significant in the final logistic regression model used for the multivariate analysis. Gender was included in the final model to control for possible confounders.

where a prevalence of $81.3 \%$ was observed for MDR-E. coli, while a prevalence of 3.2\% was recorded for ESBL-E. $\operatorname{coli}^{29}$. In contrast to our findings, a study conducted in Pakistan reported a much higher ESBL-E. coli prevalence among cattle and healthy individuals ${ }^{30}$. A related study conducted in southern Nigeria also reported a much higher ESBL and MDR-E. coli prevalence among pig SHWs ${ }^{31}$.

This study observed that the MDR-E. coli isolates recovered from SHWs were resistant to ciprofloxacin, tetracycline, ampicillin, sulfonamides, trimethoprim, and nalidixic acid. Inappropriate AMU in humans and animals has been documented in the literature to promote a selective increase in some bacterial populations as well as dissemination of resistant strains ${ }^{32,33}$. A higher ciprofloxacin resistance compared to nalidixic acid resistance was observed among MDR-E. coli isolates supporting recent reports of a similar study in southern Nigeria where a high frequency of ciprofloxacin resistance was observed in isolates recovered from pig slaughterhouse workers $^{31}$. A possible explanation for this observation is likely due to $q n r$ genes where qnrS1 is the most common in Africa especially since fluoroquinolone resistance in different regions have different characteristics ${ }^{34}$. This is not surprising as ciprofloxacin is one of the most commonly prescribed quinolones that is affordable and available over the counter in Nigeria ${ }^{35}$. The MDR-E. coli isolates were susceptible to meropenem and imipenem consistent with recent studies conducted among pig slaughterhouse workers in southern Nigeria ${ }^{31}$. This is not surprising as these antimicrobials are normally given as intravenous injections however, resistance started to emerge in poultry and pigs in the EU with VIM and IMP genes detected in E. coli and Salmonella including a few Oxa-48 genes $^{36}$. Acquired MDR does not occur only in pathogenic bacteria but in the microflora of exposed individuals and animals. According to WHO, the important drivers for the colonization of MDR pathogens is overuse and misuse of antimicrobial drugs ${ }^{5}$ although studies have reported that occupation, diet, health, sanitation, and cultural traditions are also drivers for $\mathrm{AMR}^{37}$. This could probably explain the high prevalence of MDR-E. coli observed in the present study especially since some SHWs reported self-prescription of antibiotics, incomplete dosages, poor hygiene, and sanitation amongst others.

A related study among pig SHWs in Europe reported that the workers were more likely to be colonized by resistant bacteria if they were involved in the early slaughtering processes ${ }^{38}$. This supports our study results as butchers who are involved in these early processes were the most affected possibly because of close contact with gut content at the beginning of slaughtering especially on open slabs.

Factors associated with MDR-E. coli in the present study were age, exposure to animals at home, eating at the slaughterhouse while working, collecting waste, and hand hygiene. While the former are independent risk factors, the latter is a protective factor. A related study done in Nigeria among poultry workers reported that a history of diarrheoa and work exposure of over 10 years were risk factors for feacal carriage of MDR-E. coli $i^{9}$ however the present study did not observe any association with these factors among SHWs. A similar study done among poultry SHWs confirms that some risks do exist from occupational exposure for the acquisition of MDR-E. coli $i^{39}$.

Washing hands with soap was found to be a protective factor against faecal carriage of MDR-E. coli among the workers. According to WHO, lack of clean water and sanitation facilitates the spread of antimicrobial-resistant microbes ${ }^{5}$. It has been documented that washing hands with soap and clean water is very effective in removing faecal bacteria from the hands ${ }^{40}$ confirming our study results. The importance of hand hygiene and sanitation should be encouraged in the fight against AMR.

The mechanism of AMR spread from animals to humans is not clear however, literature shows that animals are reservoirs for $E$. coli found in humans ${ }^{41}$. Our results show that SHWs were colonized by MDR-E. coli resistant to commonly used antibiotics in humans and livestock. In addition, we identified slaughterhouse waste collection as a risk factor for the acquisition of MDR-E. coli among SHWs. Poor sanitary slaughterhouse environment ${ }^{31,42}$ and occupational exposure to food animals may have been responsible for this observation. There is a paucity of studies on risk factors for MDR-E. coli in the target population. Some studies reported travel history and diet as risk factors for faecal carriage of MDR-E. coli however the present study did not consider these factors ${ }^{10,43}$.

There are potential limitations in this study that could be addressed in future research. First, the study focused on the phenotypic characterization of the E. coli isolates to determine the resistance profile of the slaughterhouse workers. Secondly, the study determined work exposure factors as well as the antimicrobial use history of the slaughterhouse workers that put them at risk of being colonized with MDR E. coli. Future studies could explore the possible origin of MDR observed among the slaughterhouse workers as well as the genomic characterization of the isolates to identify the genes, determine clonality and perform cluster analysis for traceability. 
Apparently healthy SHWs could harbor and shed MDR E. coli in their stool hence posing as a public health hazard to the general population. Prevalence of resistant $E$. coli was highest among butchers. Risk factors for faecal carriage of MDR E. coli were age, animal exposure at home, eating at work, and waste collection. Handwashing with soap and clean water was protective against acquiring MDR E. coli among the workers. This study provides additional evidence demonstrating the occupational hazards for people working in slaughterhouses. We recommend that competent authorities organize regular AMR awareness programs targeted at SHWs to mitigate public health risks. Hand hygiene and responsible use of antibiotics among SHWs should be encouraged.

\section{Methods}

Study area. This study was conducted in two major cities; Abuja metropolis, North-Central part of Nigeria $\left(9.01145^{\circ} \mathrm{N}, 7.57961^{\circ} \mathrm{E}\right)$ with a population of approximately $3 \mathrm{M}$ people and Lagos city, South-Western Nigeria $\left(6.64983^{\circ} \mathrm{N}, 3.32023^{\circ} \mathrm{E}\right)$ inhabited by $14 \mathrm{M}$ people, respectively.

Study design. A cross-sectional study approach was applied among 118 apparently healthy workers who were randomly selected from the two largest slaughterhouses situated in Abuja (84 samples) and Lagos (34 samples) from July to December 2020. All SHWs aged $\geq 18$ years, who volunteered were enrolled as study participants. All study participants signed the informed consent form explaining the purpose and benefits of the study before sample collection. An open data kit (ODK) installed on a smartphone was used to administer a structured interviewer-administered questionnaire to SHWs for the data collection on demographic characteristics and exposure factors.

Sampling method. The largest slaughterhouses each in Abuja and Lagos were selected based on the daily volume of livestock slaughter. Using a simple random sampling method, we selected workers from both slaughterhouses for the study. We collected freshly passed stool samples (one sample per worker) using sterile stool containers and transported the samples in cool boxes to the National Reference Laboratory (NRL), Nigeria Centre for Disease Control, Abuja. The samples collected in Abuja were processed within $3 \mathrm{~h}$ of sample arrival in the laboratory for the presence of $E$. coli. Samples collected in Lagos were stored at $-80^{\circ} \mathrm{C}$ thereafter they were transported to the NRL, Abuja for processing. Positivity was determined as the presence of MDR-E. coli in a stool sample.

Bacteriological analysis. Isolation of E. coli isolates. Briefly, we inoculated one gram of stool sample in $9 \mathrm{ml}$ of buffered peptone water (enrichment broth) and incubated at $37^{\circ} \mathrm{C}$ for $24 \mathrm{~h}$. Thereafter, a $10 \mu \mathrm{lloop}$-full culture from buffered peptone water was streaked onto the selective media, MacConkey agar, and incubated at $37^{\circ} \mathrm{C}$ for $24 \mathrm{~h}$. Three well-isolated suspected $\mathrm{E}$. coli colonies were streaked, usually pink to red onto the indicative media, Eosin Methylene Blue agar, and incubated at $37^{\circ} \mathrm{C}$ under aerobic conditions for $24 \mathrm{~h}^{20}$. Elevated, moist colonies with a greenish metallic sheen, suggestive of $E$. coli, were sub-cultured onto the non-selective media, Trypticase Soy agar plates, and incubated for $24 \mathrm{~h}$ at $37^{\circ} \mathrm{C}$ for the isolation of pure cultures. Conventional biochemical tests were performed on all $E$. coli isolates ${ }^{20}$ and confirmed using a commercially available biochemical test strip, Microbact GNB 24E (Oxoid, UK), according to the Manufacturer's instructions.

Antimicrobial susceptibility testing of E. coli isolates. The Kirby Bauer disk diffusion method was applied to perform antimicrobial susceptibility testing of the $E$. coli isolates according to the Clinical and Laboratory Standards Institute (CLSI, 2020) recommendations ${ }^{21}$. A panel of 16 different antibiotics of 12 classes was applied including ampicillin $(10 \mu \mathrm{g})$, azithromycin $(15 \mu \mathrm{g})$, tetracycline $(30 \mu \mathrm{g})$, gentamicin $(10 \mu \mathrm{g})$, chloramphenicol $(30 \mu \mathrm{g})$, colistin $(10 \mu \mathrm{g})$, sulfonamides $(300 \mu \mathrm{g})$, trimethoprim $(5 \mu \mathrm{g})$, cefoxitin $(30 \mu \mathrm{g})$, ciprofloxacin $(5 \mu \mathrm{g})$, nalidixic acid $(30 \mu \mathrm{g})$, nitrofurantoin $(300 \mu \mathrm{g})$, imipenem $(10 \mu \mathrm{g})$, meropenem $(10 \mu \mathrm{g})$, ceftazidime $(30 \mu \mathrm{g})$, and cefotaxime $(30 \mu \mathrm{g})$. For quality control, we used E. coli ATCC 25922 . In this study, we defined MDR as the bacteria isolate being resistant to three or more classes of antibiotics commonly used in medical and veterinary practice in Nigeria.

Multiple antibiotic resistance (MAR) indexes. We calculated the MAR index for each $E$. coli isolate using the formula MAR $=a / b$, where ' $a$ ' was the number of antibiotics that the isolate was resistant to while ' $b$ ' was the total number of antibiotics that the isolates were subjected to ${ }^{22}$.

ESBL phenotype detection by disk diffusion. Presumptive extended-spectrum beta-lactamase-producing E. coli (ESBL-E. coli) isolates were further confirmed using the disk diffusion method according to CLSI $2020^{21}$ testing for synergy applying the following disks: ceftazidime $(30 \mu \mathrm{g})(\mathrm{CAZ})$, ceftazidime + clavulanic acid $(30 / 10 \mu \mathrm{g})$ $(\mathrm{CAC})$, cefotaxime $(30 \mu \mathrm{g})(\mathrm{CTX})$, cefotaxime + clavulanic acid $(30 / 10 \mu \mathrm{g})(\mathrm{CEC})$. Following growth, the zonediameters were measured and recorded. An increase in the zone diameter by $\geq 5 \mathrm{~mm}$ around the disks containing cephalosporin with the inhibitor over the disks containing solely cephalosporin indicates ESBL production (synergy). Controls: E. coli ATCC 25929 and K. pneumoniae ATCC 700603 were used as negative and positive controls, respectively.

Risk factor survey. We administered electronic questionnaires to SHWs using ODK collect app on a smartphone to determine the risk factors for MDR E. coli colonization. We assessed the association between demographics, AMU, exposure factors, and acquiring MDR-E. coli. We obtained permission from slaughterhouse 
management before administering the questionnaire to the workers. We pretested the questionnaire at the DeiDei slaughterhouse, however; we did not include data obtained from the pretesting in the final analysis.

Statistical analysis. The data collected with ODK collect app was exported as a Microsoft Excel spreadsheet to a computer using the ODK briefcase tool. Data were analyzed using Epi Info version-7 by computing frequencies and proportions. To identify risk factors for MDR-E. coli, we performed bivariate analysis using odds-ratios (OR) as a measure of association. Risk factors identified as statistically significant at bivariate analysis were included in an unconditional logistic regression model used for multivariate analysis at a 5\% significance level. All relevant data are within the paper and available as supporting information (Supplementary Information 1).

Ethics approval. We obtained ethical approval from the Federal Capital Territory (FCT) Health Research Ethics Committee (Approval Number: FHREC/2020/01/40/04-05-20) and sought permission from the management of each slaughterhouse before the study commenced. All study participants signed the written informed consent form detailing the purpose and benefit of the study before sample collection. The study participants were also assured of confidentiality of the information obtained. All procedures were performed in accordance with the ethics committee's guidelines and requirements.

\section{Data availability}

All data generated or analyzed during this study are included in this published article [and its supplementary information files].

Received: 15 February 2021; Accepted: 16 June 2021

Published online: 25 June 2021

\section{References}

1. Kariuki, S. \& Dougan, G. Antibacterial resistance in sub-Saharan Africa: An underestimated emergency. Ann. N. Y. Acad. Sci. 1323, 43-55 (2014).

2. Katakweba, A. A. S. et al. First report on a randomized investigation of antimicrobial resistance in fecal indicator bacteria from livestock, poultry, and humans in Tanzania. Microb. Drug Resist. 24, 260-268 (2018).

3. Dadgostar, P. Antimicrobial resistance: Implications and costs. Infect. Drug Resist. 12, 3903-3910 (2019).

4. Bernabé, K. J., Langendorf, C., Ford, N., Ronat, J. B. \& Murphy, R. A. Antimicrobial resistance in West Africa: A systematic review and meta-analysis. Int. J. Antimicrob. Agents 50, 629-639 (2017).

5. World Health Organization. Antimicrobial resistance. WHO fact sheets accessed online at: https://www.who.int/news-room/factsheets/detail/antimicrobial-resistance (2020).

6. Yamaji, R. et al. A population-based surveillance study of shared genotypes of Escherichia coli isolates from retail meat and suspected cases of urinary tract infections. mSphere 3, 20 (2018).

7. Saliu, E. M., Vahjen, W. \& Zentek, J. Types and prevalence of extended-spectrum beta-lactamase-producing Enterobacteriaceae in poultry. Anim. Health Res. Rev. 18, 46-57 (2017).

8. Sebsibe, M. A. \& Asfaw, E. T. Occurrence of multi-drug resistant Escherichia coli and Escherichia coli O157:H7 in meat and swab samples of various contact surfaces at abattoir and butcher shops in Jimma town, southwest district of Ethiopia. Infect. Drug Resist. 13, 3853-3862 (2020).

9. Aworh, M. K., Kwaga, J., Okolocha, E., Mba, N. \& Thakur, S. Prevalence and risk factors for multi-drug resistant Escherichia coli among poultry workers in the Federal Capital Territory, Abuja, Nigeria. PLoS One 14, 0225379 (2019).

10. Hu, Y., Matsui, Y. \& Riley, W. L. Risk factors for fecal carriage of drug-resistant Escherichia coli: A systematic review and metaanalysis. Antimicrob. Resist. Infect. Control 9, 31 (2020).

11. Szmolka, A. \& Nagy, B. Multidrug resistant commensal Escherichia coli in animals and its impact for public health. Front. Microbiol. 4, 258 (2013).

12. Savin, M. et al. Antibiotic-resistant bacteria and antimicrobial residues in wastewater and process water from German pig slaughterhouses and their receiving municipal wastewater treatment plants. Sci. Total Environ. 727, 138788 (2020).

13. Neyra, R. C., Vegosen, L., Davis, M. F., Price, L. \& Silbergeld, E. K. Antimicrobial-resistant bacteria: An unrecognized work-related risk in food animal production. In Safety and Health at Work, vol 3 85-91 (Elsevier, 2012).

14. Abdullahi, A. et al. Occupational hazards among the abattoir workers associated with non-compliance to the meat processing and waste disposal laws in Malaysia. Risk Manag. Healthc. Policy 9, 157-163 (2016).

15. Adesokan, H. K., Akanbi, I. O., Akanbi, I. M. \& Obaweda, R. A. Pattern of antimicrobial usage in livestock animals in SouthWestern Nigeria: The need for alternative plans. Onderstepoort J. Vet. Res. 82, 1-6 (2015).

16. Ibrahim, A., Junaidu, A. \& Garba, M. Multiple antibiotic residues in meat from slaughtered cattle in Nigeria. Internet J. Vet. Med. 8, 6-10 (2012).

17. Igwe, J., Olayinka, B., Ehnimidu, J. \& Onaolapo, J. Virulent characteristics of multidrug-resistant E. coli from Zaria, Nigeria. Clin. Microbiol. Open Access 5, 20 (2016).

18. Alhaji, N. B. \& Baiwa, M. Factors affecting workers' delivery of good hygienic and sanitary operations in slaughterhouses in northcentral Nigeria. Sokoto J. Vet. Sci. 13, 29-37 (2015).

19. Nigeria Centre for Disease Control. Antimicrobial Use and Resistance in Nigeria: Situation Analysis and Recommendations. 1-158 (2017). https://ncdc.gov.ng/themes/common/docs/protocols/56_1510840387.pdf.

20. Bhoomika, S. S., Patyal, A. \& Gade, N. E. Occurrence and characteristics of extended-spectrum $\beta$-lactamases producing Escherichia coli in foods of animal origin and human clinical samples in Chhattisgarh, India. Vet. World 9, 996-1000 (2016).

21. Clinical and Laboratory Standards Institute. CLSI M100 30th Edition. J. Serv. Mark. 30, 4 (2020).

22. Sandhu, R., Dahiya, S. \& Sayal, P. Evaluation of multiple antibiotic resistance (MAR) index and Doxycycline susceptibility of Acinetobacter species among inpatients. Indian J. Microbiol. Res. 3, 299 (2016).

23. Adesoji, A. T. \& Liadi, A. M. Antibiogram studies of Escherichia coli and salmonella species isolated from diarrheal patients attending malam mande General Hospital Dutsin-ma, Katsina State, Nigeria. Pan Afr. Med. J. 37, 1-13 (2020).

24. Shitu, S., Gambo, B. A., Musa, M. O., Abubakar, A. A. \& Attahiru, M. Prevalence of multidrug-resistant Escherichia coli in suspected cases of urinary tract infection among patients attending Ahmadu Bello University Medical Center, Zaria. UMYU J. Microbiol. Res. 5, 123-130 (2020).

25. Zhuge, X. et al. Population structure and antimicrobial resistance traits of Avian-origin mcr-1-positive Escherichia coli in Eastern China, 2015-2017. Transboundary and Emerging Diseases. 66, 1920-1929 (2019). 
26. Landers, T. F., Cohen, B., Wittum, T. E. \& Larson, E. L. A review of antibiotic use in food animals: Perspective, policy, and potential. Public Health Rep. 127, 4-22 (2012).

27. Van, T. T. H., Yidana, Z., Smooker, P. M. \& Coloe, P. J. Antibiotic use in food animals worldwide, with a focus on Africa: Pluses and minuses. J. Glob. Antimicrob. Resist. 20, 170-177 (2020).

28. Thenmozhi, S., Rajeswari, P., Suresh Kumar, B. T., Saipriyanga, V. \& Kalpana, M. Multi-drug resistant patterns of biofilm-forming Aeromonas hydrophilia from urine samples. Int. J. Pharm Sci Res. 5, 2908-2918 (2014).

29. Campbell, J. I., Nhung, N. T., Nhung, H. N., Van Minh, P. \& Wagenaar, J. A. Prevalence and risk factors for carriage of antimicrobialresistant Escherichia coli on household and small-scale chicken farms in the Mekong Delta of Vietnam. J. Antimicrobial Chemother. https://doi.org/10.1093/jac/dkv053 (2015).

30. Saleem, R., Ejaz, H., Zafar, A. \& Younas, S. Phenotypic characterization of extended-spectrum-beta-lactamase producing E. coli from healthy individuals, patients, sewage sludge, cattle, chickens, and raw meat. Pak. J. Med. Sci. 33, 886 (2017).

31. Egbule, O. S. \& Iweriebor, B. C. Beta-Lactamase-producing Escherichia coli isolates recovered from pig handlers in retail shops and Abattoirs in selected localities in Southern Nigeria: Implications for public health. Antibiotics. 10, 1-10 (2021).

32. Singh, S., Shukla, S., Tandia, N., Kumar, N. \& Paliwal, R. Pharma Sci. Monit. 5, 184-197 (2014).

33. Daniels, J. B. et al. Role of ceftiofur in selection and dissemination of blaCMY-2-mediated cephalosporin resistance in salmonella enterica and commensal Escherichia coli isolates from cattle. Appl. Environ. Microbiol. 75, 3648-3655 (2009).

34. Qin, T.-T. et al. SOS response and its regulation on the fluoroquinolone resistance. Ann. Transl. Med. 3, 358 (2015).

35. Suleiman, I. A. \& Tayo, F. Comparative costs of antibacterial usage in sexually transmitted infections in a Nigerian teaching hospital. Trop. J. Pharm. Res. 9, 549-555 (2010).

36. Nordmann, P., Naas, T. \& Poirel, L. Global spread of carbapenemase- producing enterobacteriaceae. Emerg. Infect. Dis. 17, 1791$1798(2011)$

37. Hendriksen, R. S. et al. Global monitoring of antimicrobial resistance based on metagenomics analyses of urban sewage. Nat. Commun. 10, 20 (2019).

38. Dohmen, W. et al. ESBL carriage in pig slaughterhouse workers is associated with occupational exposure. Epidemiol. Infect. 145, 2003-2010 (2017).

39. van den Bogaard, A. E. Antibiotic resistance of faecal Escherichia coli in poultry, poultry farmers and poultry slaughterers. J. Antimicrob. Chemother. 47, 763-771 (2001).

40. Burton, M. et al. The effect of handwashing with water or soap on bacterial contamination of hands. Int. J. Environ. Res. Public Health 8, 97-104 (2011).

41. Founou, L. L., Founou, R. C. \& Essack, S. Y. Antibiotic resistance in the food chain: A developing. Front. Microbiol. 7, 1-19 (2016).

42. De, N., Ayobami, B. O. \& Bosede, A. Microbiological assessment of agege abattoir situated in Lagos State, Nigeria. IOSR J. Environ. Sci. Ver. I(9), 2319-2399 (2015).

43. Huijbers, P. M. C. et al. Extended-spectrum and AmpC $\beta$-lactamase-producing Escherichia coli in broilers and people living and/or working on broiler farms: Prevalence, risk factors, and molecular characteristics. J. Antimicrob. Chemother. 69, 2669-2675 (2014).

\section{Acknowledgements}

The authors would like to appreciate Dr. Pernille Nilsson of Denmark Technical University and Prof. Iruka Okeke of the University of Ibadan, Nigeria for their contributions towards the completion of this research. We would like to acknowledge the support of the Chief Veterinary Officer of Nigeria, Dr. Alabi Olaniran, and Dr. Peter Umanah at the Department of Veterinary \& Pest Control Services, Federal Ministry of Agriculture and Rural Development, Abuja. The authors appreciate Dr. Chikwe Ihekweazu, Director General, Nigeria Center for Disease Control, Abuja for providing laboratory support towards this research at the National Reference Laboratory (NRL), Abuja. We acknowledge the intellectual contributions of Miss Eme Ekeng, Mr. Micheal Popoola, and Mr. Akinpelu Muftan of NRL, Abuja towards the success of this research. The authors appreciate the support of Dr. Stephen Okeme at Karu Slaughterhouse, Abuja, and Dr. Ayokunle Omileye at Oko-oba Agege Slaughterhouse, Lagos.

\section{Author contributions}

R.H. and M.A. made substantial contributions to conception and design. M.A. was the principal investigator, designed data collection tools, collected data, conducted laboratory work, analyzed and interpreted the data. D.A. and B.H. assisted in designing the data collection tool. D.A. supported with data collection. R.H. and N.M. supervised the laboratory aspect of the research. M.A. wrote the first draft of the manuscript. R.H., B.H., and N.M. revised the article critically for important intellectual content. All authors read and approved the final manuscript.

\section{Competing interests}

The authors declare no competing interests.

\section{Additional information}

Supplementary Information The online version contains supplementary material available at https://doi.org/ 10.1038/s41598-021-92819-3.

Correspondence and requests for materials should be addressed to M.K.A.

Reprints and permissions information is available at www.nature.com/reprints.

Publisher's note Springer Nature remains neutral with regard to jurisdictional claims in published maps and institutional affiliations. 
(c) (i) Open Access This article is licensed under a Creative Commons Attribution 4.0 International cc) License, which permits use, sharing, adaptation, distribution and reproduction in any medium or format, as long as you give appropriate credit to the original author(s) and the source, provide a link to the Creative Commons licence, and indicate if changes were made. The images or other third party material in this article are included in the article's Creative Commons licence, unless indicated otherwise in a credit line to the material. If material is not included in the article's Creative Commons licence and your intended use is not permitted by statutory regulation or exceeds the permitted use, you will need to obtain permission directly from the copyright holder. To view a copy of this licence, visit http://creativecommons.org/licenses/by/4.0/.

(C) The Author(s) 2021 